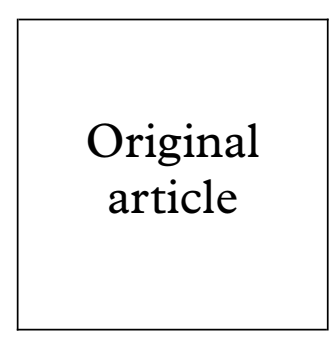

\author{
Monica Mansell-Gregory, Barbara Romanowski
}

\title{
Randomised double blind trial of EMLA for the control of pain related to cryotherapy in the treatment of genital HPV lesions
}

Objective: To evaluate the efficacy of EMLA (eutectic mixture of local anaesthetic) for the con-
trol of pain related to cryotherapy for the treatment of human papilloma virus (HPV) in the out-
patient setting.
Design: A randomised, double blind, placebo controlled study.
Subjects: 40 patients $(20$ male and 20 female) with external genital HPV involving an area of no
greater than $1 \mathrm{~cm}^{2}$.
Setting: The Capital Health Sexually Transmitted Disease Clinic, Edmonton, Alberta, Canada.
Results: Patients in whom EMLA was utilised reported significantly lower scores than those in
the control group. Women reported pain scores of $4.7 / 10$ and $0.9 / 10$ in placebo and EMLA
groups respectively (p $<0.01)$. Men reported pain scores of $6.4 / 10$ and $3.1 / 10$ in placebo and
EMLA groups respectively $(\mathrm{p}<0.01)$. Men reported significantly higher pain scores than women
in the EMLA groups $(\mathrm{p}<0.01)$. There was no significant difference in pain scores between men
and women in the placebo groups. Conclusion: EMLA can provide efficacious topical anaesthesia before cryotherapy for the treatment of external genital HPV.

(Sex Transm Inf 1998;74:274-275)

Keywords: human papillomavirus; EMLA; cryotherapy

\section{Introduction}

Human papillomavirus (HPV) is a prevalent sexually transmitted infection. It has been associated with an increased risk of both cervical and anogenital carcinoma. ${ }^{12}$ Treatment is aimed at eradicating the physical presentation of condyloma acuminatum. Cryotherapy, an effective commonly utilised form of therapy, is painful and often requires multiple visits.

Until recently, topical anaesthesia has been difficult, since most dermal anaesthetic agents are unable to penetrate deeply enough to produce effective anaesthesia. While good anaesthesia is available via subcutaneous infiltration, the procedure of injecting anaesthetic could be arguably as traumatic as the treatment itself and is therefore not a viable option. Recent pharmacological developments have produced a topical anaesthetic that is well absorbed without the need for subcutaneous infiltration.

EMLA (eutectic mixture of local anaesthetic, Astra Pharmaceuticals) composed of lignocaine $2.5 \%$ and prilocaine $2.5 \%$, has been shown to be an effective topical anaesthetic for invasive genital procedures such as punch biopsies and electrocoagulation. ${ }^{3}$ EMLA has also been shown to be efficacious in pain reduction for HPV laser therapy and pain related to cryotherapy. ${ }^{4-7}$ Anaesthesia can be obtained in the genital region within 5-15 minutes with peak plasma concentrations occurring 20-45 minutes after application. Common side effects include blanching, erythema, and an initial burning sensation. EMLA is considered safe and is available without a prescription in Canada. ${ }^{8}$
The objective of this study was to determine whether pain induced by cryotherapy can be decreased or controlled using EMLA.

\section{Patients and methods}

Appropriate patients attending the Capital Health STD Clinic for treatment of genital warts were approached to participate if they met the following inclusion/exclusion criteria: external condylomata acuminata involving the labia minora introitus, penile shaft, or glans having a total surface area of no greater than 1 $\mathrm{cm}^{2}$; general good health; between the ages of 16 and 35 years; and written informed consent before participation. The exclusion criteria were as follows: other pathological lesions or rashes; idiopathic genital pain; history of ethanol, street drug, narcotic, or analgesic use in the preceding 24 hours; use of antidepressants in the preceding week; concurrent use of sulpha drugs; presence of cardiac disease; or prior participation in this trial. People on sulpha drugs were excluded because of the remote possibility of methoglobinaemia. People on antidepressants were eliminated due to the known analgesic enhancement of antidepressants. People with lesions outside the labia minora, introitus, shaft, and glans penis were eliminated to reduce the bias by studying only genital areas with a minimally keratinised, highly vascular epidermis. The study was approved by the ethics review committee at the University of Alberta.

Patients participating in the trial had visible acetowhite HPV lesions which were measured and recorded by the investigating nurse. The investigating nurse then left the room and a 


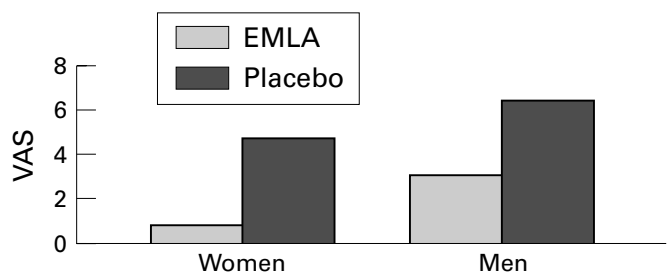

Figure 1 Mean visual analogue scores for pain. The VAS (visual analogue scale) was modified to include numeric anchors from 0 to 10 . A rating of 0 denotes no pain at all. $A$ rating of 10 denotes the greatest pain ever experienced.

second nurse applied either EMLA or unscented Keri lotion (placebo) to the lesion(s) utilising a list from a randomisation table. Keri lotion is indistinguishable from EMLA under the conditions of this study. A generous application of the cream was left on for 10 minutes. Liquid nitrogen via a spray canister was applied by the investigating nurse as three freeze thaw cycles. Immediately after the treatment, the patients were asked to rate their pain using a modified visual analogue scale (VAS). ${ }^{910}$ The VAS was supplemented with numeric anchors from 0 to 10 to provide the greatest simplicity for study participants. A score of zero represented no pain and a score of 10 represented the greatest pain. Patients were also asked to provide a verbal pain description of the treatment experience.

A patient survey was conducted to determine if the additional time required for anaesthesia would be acceptable to this patient population should EMLA prove efficacious in this setting.

Statistical analysis was done using independent measures ANOVA hypothesis testing on MS Excel from Microsoft Word. Comparisons were done between placebo and drug groups considering the modified VAS for males and females.

\section{Results}

Forty patients were enrolled; 20 men and 20 women. Only the drug group demonstrated rare cases of erythema, blanching, and an occasional itchy sensation at initial application. There were no serious adverse effects. The placebo and EMLA groups demonstrated a significant difference in VAS scores for pain (see fig 1). The mean VAS for women was $0.9 / 10$ and $4.7 / 10$ for EMLA and control groups respectively $(p<0.001)$. The variance for women was 2.23 and 1.65 for placebo and EMLA groups respectively. The mean VAS for men was 3.1/10 and $6.4 / 10$ for the EMLA and control groups respectively $(\mathrm{p}<0.01)$. The variance for men was 4.93 and 5.43 for placebo and EMLA groups respectively. Pain scores for men and women were compared. Men reported significantly higher pain scores in the EMLA group when compared with women $(p<0.01)$. Differences in pain scores between men and women in the control group were not significant.

Patients were also surveyed to determine if the additional time required for the application of EMLA was acceptable to this patient population. For the women surveyed, $75 \%$ stated that the additional time required to institute pain control was acceptable, $25 \%$ were indifferent, and none reported the additional time as unacceptable. For the men, $65 \%$ stated the additional time was acceptable, $30 \%$ were indifferent, and $5 \%$ reported the additional time to be unacceptable.

\section{Discussion}

Patients in this study had significantly lower pain scores when EMLA was applied 10 minutes before cryotherapy. In any pain study there is tremendous room for bias owing to the inherent subjective nature of pain. In this study design, as many confounders as possible were eliminated. Equal numbers of patients with similar lesion locations were recruited into each group. The presence of pharmaceutical enhancements was eliminated as much as possible. The VAS was modified to reduce user error. It should be noted however that the validity of any pain scoring system needs to be considered as a source of possible bias.

The difference in VAS between placebo and EMLA was markedly significant for women and men. The actual levels of pain are an interesting finding. Men report significantly higher pain scores when compared with women in the EMLA group. The anatomical regions studied in men were less mucosal than those studied in women and consequently, the absorption of anaesthetic through the epidermis would be less efficient. Additional application time of EMLA for men in this setting may be warranted. This however requires further investigation.

\section{Conclusion}

EMLA can be used to provide efficacious topical anaesthesia before cryotherapy for the treatment of external genital HPV. Side effects are minimal and the drug is well tolerated. The additional waiting time required appears to be acceptable to the majority of patients surveyed. These encouraging results based on a relatively small sample size should be confirmed in a larger study.

We thank Sharon Hill, Sharyn Hewitt, and the staff at the STD clinic for their vital contributions to this study; also Dr Sheila Mansell for her work on the statistical analysis of this study.

1 Franco E. Epidemiology of anogenital warts and cancer. Obstetrics and gynecology clinics of North America: human papilloma virus I. Toronto: WB Saunders, 1996;23:597-617. 2 Ferenczy A. Epidemiology and clinical pathophysiology of condyloma acuminata. Am $\mathcal{F}$ Obstet Gynecol 1995;68:162-5.

3 Berg G, Lillieborg S, Stoltz E. Lidocaine/prilocaine cream (EMLA $®)$ versus infiltration anesthesia: a comparison of analgesic efficacy for punch biopsy and electrocoagulation of genital warts in men. Genitourin Med 1992;68:162-5.

4 Lassus A, Kartamaa M, Happonen H. A comparative study of topical anesthesia with lidocaine/prilocaine cream (EMLA) for laser surgery of genital warts in men. Sex Transm Dis 1990;17:130-2.

5 Rylander E, Sjoberg I, Lillieborg S, et al. Local anaesthetic of the genital mucosa with lidocaine/prilocaine cream (EMLA) for laser treatment of condylomata acuminata: a placebo controlled study. Obstet Gynecol 1990;75:302-6.

6 Menter A, Black-Noller G, Riendeau L, et al. The use of Menter A, Black-Noller G, Riendeau $L$, et al. The use of
EMLA cream and $1 \%$ lidocaine infiltration in men for the relief of pain associated with the removal of genital warts in cryotherapy. F Am Acad Dermatol 1997;37:96-100.

7 Buckley M, Benfield P. Eutectic lidocaine/prilocaine cream a review of the topical anaesthetic/analgesic efficacy of a eutectic mixture of local anaesthetics (EMLA) Drugs 1993; 46:127-9, 143-4.

8 EMLA. Product monograph, 1-3, 19. Mississauga, Ontario: Astra Drug Company, November 1993.

9 Mitchell M, Portenoy R, Laska E. The design of analgesic clinical trials. In: Advances in pain research and therapy. Vol 18. New York: Raven Press, 1991:59-61.

10 Ramamurthy S, Rogers J. Decision making in pain management. St Louis: Mosby Year Book, 1993:12-14. 